\title{
Module Optical Analyzer: Identification Of Defects On The Production Line
}

\author{
Rebeca Herrero, Stephen Askins , Ignacio Antón , Gabriel Sala , Kenji Araki \\ Hirokazu Nagai
}

\begin{abstract}
The usefulness of the module optical analyzer when identifying module defects on production line is presented in this paper. Two different case studies performed with two different kind of CPV modules are presented to show the use of MOA both in IES-UPM and Daido Steel facilities.
\end{abstract}

\section{INTRODUCTION}

The module optical analyzer (MOA) is a device developed by the Instituto de Energía Solar for the optical-angular characterization of a concentrator photovoltaic (CPV) module. It is based on the luminescence inverse (LI) method [1], which consists of forward biasing the module to evaluate the emitted light to obtain the angular transmission functions of each optical unit of the module, and their angular deviations. While both the basis of the LI method and first implementations of the MOA equipment have already been presented [2], [3], in this work we study the performance of the MOA in a real CPV production line. In particular, we show two different case-study of how with the instrument has been used in a high volume manufacturing scenario to gather useful information of modules before installation.

\section{MOA AND LI METHOD}

The measurement scheme of the MOA system is based on the LI method (FIGURE 1). The module is forward biased at dark conditions to reproduce a Lambertian and uniform emission at the multi-junction (MJ) solar cell. The forward biasing of a CPV module is not free of risk [4]. Thus, the direct current values recommended for the LI method correspond to $10 \%$ of the value of the working photocurrent at which the CPV module is designed to perform best. A concentrating optics with large aperture area (as the collimator mirror of the Helios 3198 solar simulator [5]) must be included in the measurement to distinguish between the angular directions of the module emission at its focal plane. If some kinds of image-formation optical systems are placed at the focal plane of the already mentioned collimator mirror, not only the angular but also the spatial properties of the module emission can be investigated. The assembly formed by the optical systems and sensor at which the images of the module are formed is what we call the MOA system.

In the LI method, as established by the reversibility principle [6], the emission of each optical system-cell unit comprising the module is transmitted through the concentrator optical system up to its input aperture. This emission fills all possible directions the light can follow when illuminating the module from its input aperture which lead to the 2-dimensional (2D) impulse-response angular transmission function $\mathrm{H}(\alpha, \phi)$. The angular transmittance function of a concentrator under a particular angular light distribution $\mathrm{S}(\alpha, \phi)$ is obtained by the convolution between the impulse-response function and the light source as

$$
T_{\text {source }}(\alpha, \phi)=H(\alpha, \phi) \otimes S(\alpha, \phi)
$$

where a and $f$ are the zenith and azimuth angular directions. 


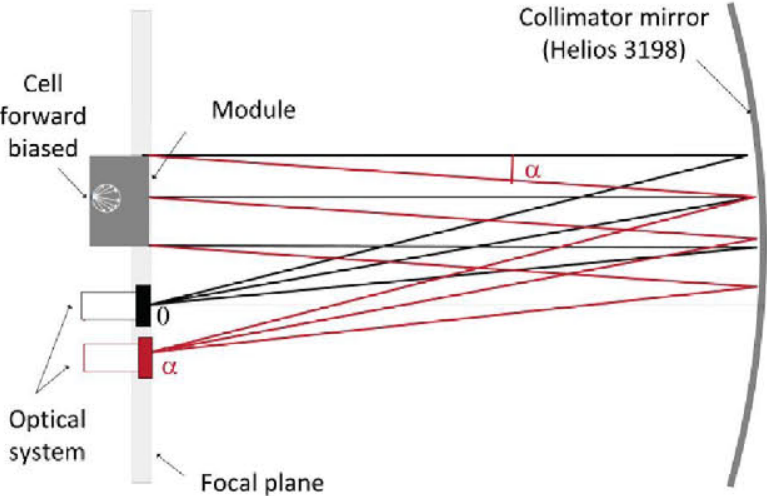

FIGURE 1. Measurement scheme of the luminescenceinverse (LI) method including the module optical analyzer (MOA)

From the angular transmittance function, the most common figure of merit which is the acceptance angle (defined as the angular direction at which the function reaches $90 \%$ of its maximum) can be obtained. From the angular transmittance function, it is also possible to obtain the optimum alignment of each unit (defined as the angle at which the angular transmittance function is maximized). This is very valuable information as misalignments between units are usually the most dominant factor for power loss at tracker level due to the narrowing effect they have in the angular transmittance of CPV modules.

\section{MOA PERFORMANCE ON THE PRODUCTION LINE}

A prototype of MOA for a production line was developed and installed at Daido Steel Co facilities in Nagoya (Japan) within the framework of the European Union project entitle "A new generation of concentrator photovoltaic cells, modules and systems", NGCPV (283798) [7]. In particular, a task involving the development of tools for test and quality control of modules in manufacturing line was accomplished through a collaborative research between the Japanese company Daido Steel and the IES-UPM. The modules to be tested are the so-called "Intrepid" modules (Dome-Khöler [8]) which are also developed in parallel in the same project, and the conventional Daido modules (Dome-pyramid).

A highly accurate alignment control at the time of installation is needed to reduce the power loss at tracker level for modules with narrow acceptance angles. Instead of performing this kind of quality control which can be very difficult and costly, a proper quality control in the factory is recommended to limit the misalignments amongst the module units, and consequently maximizing its acceptance angle.
Two different case studies are presented in this paper to show how the MOA performs quality controls of the optical-angular properties of CPV modules. The first case of study has been performed at the IESUPM, and it is based on the characterization of "Intrepid" modules before being installed at the IESUPM experimental field in Madrid. The second case of study has been performed at Daido Steel facilities, and it consists of evaluation of modules (with new vendor components) on the production line.

\section{MOA PERFORMANCE AT IES-UPM}

Before their installation at the tracker, 22 out of 50 CPV modules randomly selected were characterized by the MOA system at the IES-UPM. This characterization was performed after concluding (by conventional measurements at the solar simulator) that the modules acceptance angles were lower than the one of the individual optical system-cell unit.

Data provided by the MOA system consist of images of the module emitting light at different angular directions. The light emitted by each unit in the module can be quantified and plotted as function of the angular direction of emission to obtain the angular transmittance functions of the units comprising the module.

The MOA system installed at IES-UPM is able to investigate the optical-angular properties of the module in any direction. Nevertheless, both horizontal (X direction) and vertical ( $\mathrm{Y}$ direction) are chosen in this study as they are the most common directions investigated by direct methods. 


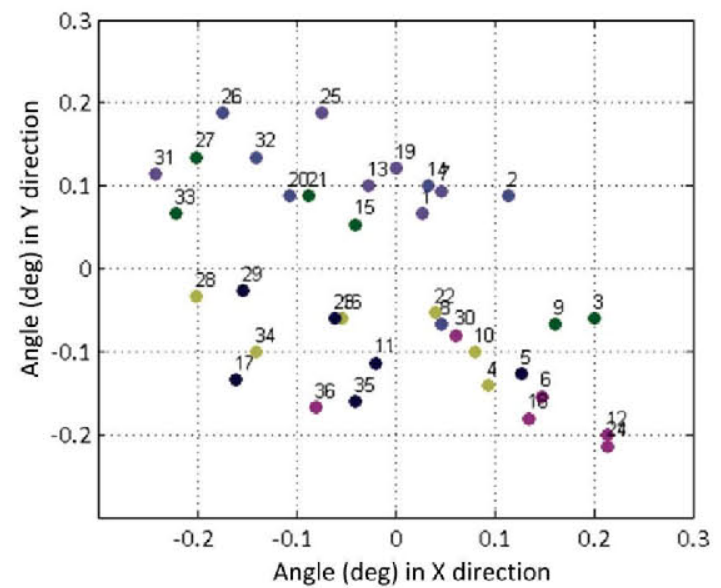

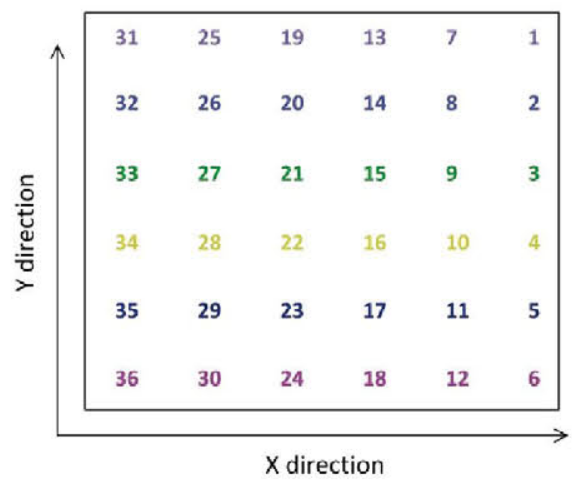

FIGURE 2. (Top) Misalignments between units in an "Intrepid" CPV module measured by MOA; (Bottom) Units position at the "Intrepid" module at front view.

The acceptance angles of the "Intrepid" modules measured by MOA were confirmed to be 0.3 degrees lower in average if compare with the one of the individual optical system-cell unit. This decrease in angular tolerance is mainly caused by misalignments between units as already commented. When plotting the units misalignments for all the modules, a pattern coincident between them was observed. In particular for the modules under investigation, the alignment of each unit was observed to be related to the position of the unit in the module as presented in FIGURE 2.

At this moment, it must be stated that the repeatability of "Intrepid"-MOA measurements has been proven to be very high $(\mathrm{RMSE}=0.002)$ which excludes the idea of any deformation produced by the holding structure at the measurement set-up. In fact, the presence of these misalignments seems to lie in the module enclosure as the manufacturer has high confidence on receiver alignment. In fact, a slight variation in size between lens parquet and bottom plate $(0.2 \%$ smaller the bottom plate) was observed which could explain previous results.
Previous results were obtained when measuring with MOA at ambient laboratory temperature which was $20^{\circ} \mathrm{C}$. However, the module under operating conditions reaches higher temperatures in both lenses parquet and back plate of the module. This increase in temperature can involve a thermal expansion in both lenses parquet and back plate that can modify the measured misalignments at $20^{\circ} \mathrm{C}$. To observe the misalignments variation with temperature, a MOA measurement was conducted while increasing the temperature of a module to reproduce different outdoor actual conditions. Several IR bulbs placed at the back side of the module were used to vary its temperature. The module back plate that is made out of aluminum was covered with an adhesive black vinyl to increase the absorption. Several thermocouples were placed between the black cover and the back plate to confirm that the temperature distribution of this surface was rather homogeneous (with variations of $4^{\circ}$ between the center and the edges of the module). Also the temperature inside the module is measured with a thermocouple to assure the measurements are performed under steady-state conditions. The temperatures used in this experiment are close to the ones already published of the module in operation [9].

TABLE 1. Temperatures at which MOA analysis is performed

\begin{tabular}{cc}
\hline $\begin{array}{c}\text { Temperature } \\
\text { at back plate }\left({ }^{\circ} \mathbf{C}\right)\end{array}$ & $\begin{array}{c}\text { Temperature } \\
\text { inside the module }\left({ }^{\circ} \mathbf{C}\right)\end{array}$ \\
\hline 20 & 20 \\
55 & 28 \\
66 & 30 \\
71 & 36 \\
\hline
\end{tabular}

The optical-angular properties of the modules under investigation do not show any significant variation while reaching the temperatures presented in TABLE 1. It can be explained due to the differences between the thermal coefficients of the back plate and lenses parquet. The one from PMMA lenses parquet depends directly on the process the lens manufacturing. Its value is at least 4 times the one from aluminum. Thus, even the aluminum back plate reaches higher temperatures than the lens parquet, the thermal expansion is not largest.

The previous experiment was also carried out during a fast temperature increase of back plate but not of the lenses parquet. A fast MOA evaluation was performed during this transient regimen of temperatures, with temperature increase from $20^{\circ} \mathrm{C}$ to $50^{\circ} \mathrm{C}$ in back plate but without any increase in lenses parquet (at $20^{\circ}$ ). If comparing how the misalignments between units vary during this transient, it can be observed that they decrease while increasing the back plate temperature. This result is consistent with the 
idea of having a back plate $0.2 \%$ smaller than lens parquet. If the temperature of the back plate increases its size does while the lens keeps unaltered. Thus units change their pointing to the center of the module 0.03 degrees in average, as presented in FIGURE 3.

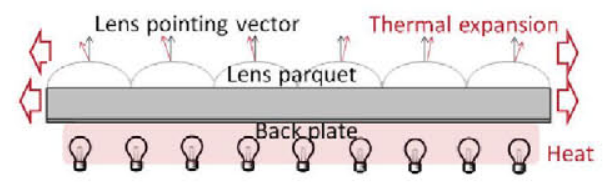

FIGURE 3. (Top) Misalignments between units are consistent with the idea of having a back plate $0.2 \%$ smaller than lens parquet; (Bottom) Misalignments between units are minimized if the temperature of the bottom plate is increased but not the one of the lenses

However, it must be pointed out that the first experiment in steady-state conditions is the one which reproduces the real outdoor performance conditions of the module and thus, there is no significant change between misalignments while varying the temperature.

\section{MOA PERFORMANCE AT DAIDO STEEL}

The design of the MOA system (FIGURE 4) developed by the IES-UPM to be installed at Daido Steel was determined by the Helios 3198 configuration already installed in the production line. The dimensions of the device are imposed by the limited space available to replace the lamp with the MOA system. Consequently, the evaluation of the opticalangular properties of the CPV module was restricted to only 1-dimension ( $Y$ direction). Nevertheless, a coarse evaluation is also performed in the $\mathrm{X}$ direction to confirm both the alignment of the MOA (if the MOA system is aligned with the module structure) and the alignments of the units (if the optimum alignment is not confirmed in a save interval of \pm 1 degrees).

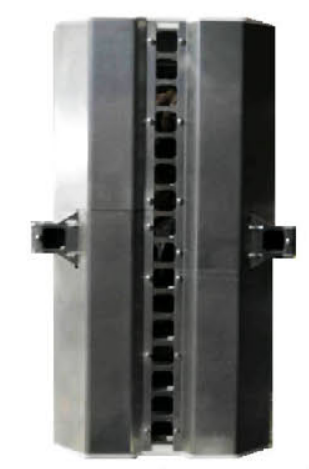

FIGURE 4. MOA system scheme at Daido Steel facilities: evaluation in 1-dimension ( $\mathrm{Y}$ direction)
The MOA installed in the factory has been able to detect and characterize the effect of real defects encountered by Daido Steel in production. The first months of MOA use on the Daido assembly line coincided with the introduction of components from a new vendor, in particular, the side-wall of the module. Modules with side-walls from a new vendor showed the same power output but a significantly reduced acceptance angle (from 0.9 to 0.69 degrees) when measured with the MOA due to misalignments between units. The reason of this enclosure deformation was identified as the position of rivet hole of the side-wall. Simple IV testing would not have detected this problem.

\section{CONCLUSIONS}

By the collaboration of company Daido Steel, the Module Optical Analyzer (MOA) has been proven to be very useful not only at laboratory level but also in production line.

The MOA evaluates the optical-angular properties of the module in short measurement times fulfilling the requirements imposed by a production line. In particular, it has been confirmed that the misalignments between units can be revealed in only few seconds, and thus it can help the manufacturer to find module defects at time before installation.

\section{ACKNOWLEDGMENTS}

This work has been partially supported by Community of Madrid under Project Numancia-2 (S2009/ENE-1477), and the European Union under Project NGCPV (FP7-ENERGY-2010-1). The MOA pending patent with number EP13382131.

\section{REFERENCES}

1. R. Herrero, C. Domínguez, S. Askins, I. Antón, and G. Sala, "Luminescence inverse method For CPV optical characterization," Opt. Express, vol. 21, no. S6, pp. A1028-A1034, Nov. 2013.

2. R. Herrero, C. Dominguez, S. Askins, I. Anton, G. Sala, and J. Berrios, "Angular Transmission Characterization of CPV Modules Based On CCD Measurements," in 6th International Conference on Concentrating Photovoltaic Systems, A. W. Betts, F. Dimroth, R. D. McConnell, and G. Sala, Eds. Melville: Amer Inst Physics, pp. 131-134.

3. R. Herrero, S. Askins, C. Dominguez, I. Anton, and G. Sala, "Equipment for Static Characterization of Angular Transmission and Misalignments of CPV," in 
7th International Conference on Concentrating

Photovoltaic Systems, F. Dimroth, S. Kurtz, G. Sala, and A. W. Bett, Eds. Melville: Amer Inst Physics.

4. Siefer, G., "Accelerated indoor aging test procedure for concentrator modules," in 21st European Photovoltaic Solar Energy Conference 2006. Proceedings. CD$R O M, 2006$, pp. 2101-2104.

5. C. Domínguez, I. Antón, and G. Sala, "Solar simulator for concentrator photovoltaic systems," Opt Express, vol. 16, no. 19, pp. 14894-14901, 2008

6. R. C. JONES, "On Reversibility and Irreversibility in Optics," J. Opt. Soc. Am., vol. 43, no. 2, pp. 138-143, Feb. 1953.

7. Datas, A., "NGCPV: A new generation of concentrator photovoltaic cells, modules and systems," in $28 t h$ European Photovoltaic Solar Energy Conference and Exhibition, EU PVSEC 2013. Proceedings. DVD-ROM, 2013, pp. 88-93.

8. P. Zamora Herranz, P. Benitez Gimenez, Y. Li, J. C. Miñano Dominguez, J. Mendes Lopes, and K. Araki, "Dome-shaped Fresnel-Köhler: a novel high performance optical concentrator," in EU PVSEC Proceedings, Frankfurt, Alemania, 2012, pp. 253-256.

9. Y. Ota, S. Tsuyoshi, N. Hirokazu, K. Araki, and N Kensuke, "Reduction of Operating Temperature in 25 Series-Connected 820X CPV," Jpn. J. Appl. Phys., vol. 52,2013 